\title{
Experimental limits on anomalous quartic gauge boson couplings at LEP
}

\section{Michela Biglietti*}

Università di Napoli "Federico II", Dipartimento di Scienze Fisiche e sezione INFN, Via Cinthia - 80126 - Napoli, Italy,

E-mail: ibiglietti@na.infn. it

ABstract: Triple boson production, $\mathrm{W}^{+} \mathrm{W}^{-} \gamma$ and $\mathrm{Z} \gamma \gamma$, is studied using the data collected at LEP2. Selected events are used to measure the energy evolution of the $\mathrm{e}^{+} \mathrm{e}^{-} \rightarrow \mathrm{W}^{+} \mathrm{W}^{-} \gamma$ and $\mathrm{e}^{+} \mathrm{e}^{-} \rightarrow \mathrm{Z} \gamma \gamma$ cross sections. The results are in agreement with Standard Model expectations. Such processes allow to set direct limits on possible anomalous contributions to the quartic gauge boson vertices $\mathrm{W}^{+} \mathrm{W}^{-} \gamma \gamma, \mathrm{W}^{+} \mathrm{W}^{-} \mathrm{Z} \gamma$ and $\mathrm{ZZ} \gamma \gamma$.

\section{Study of anomalous quartic gauge boson couplings}

The high energy LEP data offer a new insight into the Standard Model of electroweak interactions by investigating the triple production process $\mathrm{e}^{+} \mathrm{e}^{-} \rightarrow \mathrm{W}^{+} \mathrm{W}^{-} \gamma$ and $\mathrm{e}^{+} \mathrm{e}^{-} \rightarrow \mathrm{Z} \gamma \gamma$. The selected events are used to measure the cross section of the processes, within a well defined theoretical acceptance.

The $\mathrm{W}^{+} \mathrm{W}^{-} \gamma$ final state may be sensitive to the $\mathrm{W}^{+} \mathrm{W}^{-} \mathrm{Z} \gamma$ and $\mathrm{W}^{+} \mathrm{W}^{-} \gamma \gamma$ vertices, called charged quartic gauge couplings (QGC), presented in Figure 1a and Figure 1b. In addition the $\nu \bar{\nu} \gamma \gamma$ final state can also originate from the QGC diagram shown in Figure 1b.

For such processes, Standard Model quartic boson couplings are not expected to play a significant role at LEP energies. Nevertheless they are sensitive to possible anomalous contributions from physics beyond the Standard Model.

The $\mathrm{Z} \gamma \gamma$ final states could originate from the anomalous $\mathrm{s}-$ channel exchange of a $\mathrm{Z}$ boson (Figure 1c), forbidden at tree level in the Standard Model and called neutral anomalous QGC.

The contribution of anomalous QGC can be described by three additional dimension-six

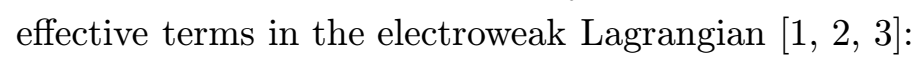

$$
\mathcal{L}^{0}=-\frac{\pi \alpha}{4 \Lambda^{2}} a_{0} F_{\mu \nu} F^{\mu \nu} \mathbf{W}_{\rho} \cdot \mathbf{W}^{\rho}
$$

${ }^{*}$ Speaker. 


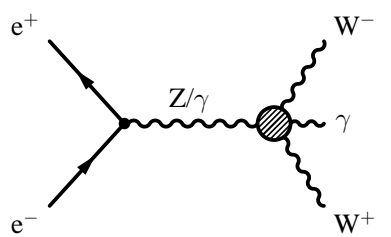

a)

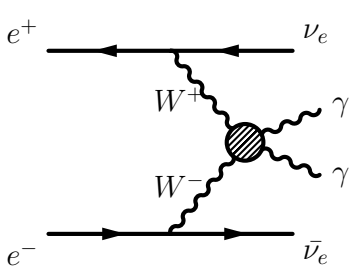

b)

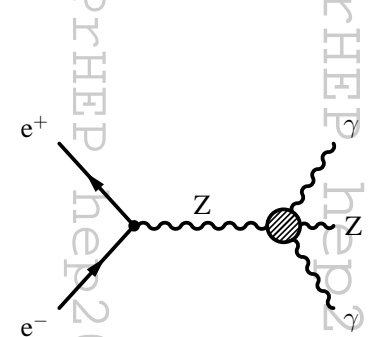

c)

Figure 1: Charged (a and b) and neutral (c) QGC Feynman diagrams.

$$
\begin{aligned}
\mathcal{L}^{c} & =-\frac{\pi \alpha}{4 \Lambda^{2}} a_{c} F_{\mu \rho} F^{\mu \sigma} \mathbf{W}^{\rho} \cdot \mathbf{W}_{\sigma} \\
\mathcal{L}^{n} & =-i \frac{\pi \alpha}{4 \Lambda^{2}} a_{n} \epsilon_{i j k} W_{\mu \alpha}^{(i)} W_{\nu}^{(j)} W^{(k) \alpha} F^{\mu \nu}
\end{aligned}
$$

where $\alpha$ is the fine structure constant, $F_{\mu \nu}$ is the field strength tensor of the photon and $\mathbf{W}_{\sigma}$ is the weak boson field. The parameters $a_{i}(i=0, c, n)$ describe the strength of the anomalous QGC and are zero in the Standard Model and $\Lambda$ represents the scale of the possible New Physics responsible for the anomalous contributions. Both $\mathcal{L}^{0}$ and $\mathcal{L}^{c}$ conserve $\mathrm{C}$ and $\mathrm{P}$ separately and generate $\mathrm{W}^{+} \mathrm{W}^{-} \gamma \gamma$ and $\mathrm{ZZ} \gamma \gamma$ couplings. The $\mathrm{CP}$ violating term $\mathcal{L}^{n}$ results in an anomalous $\mathrm{W}^{+} \mathrm{W}^{-} \mathrm{Z} \gamma$ coupling.

In the Standard Model description of the investigated process, the photon production occurs via bremsstrahlung, yielding low energy photons preferentially produced close to the beam direction. The production due to QGC results instead in a harder energy spectrum and a more central angular distribution of the photons. Theoretical predictions for anomalous QGC are obtained with the EEWWG, EENUNUGANO and EEZGG analytic

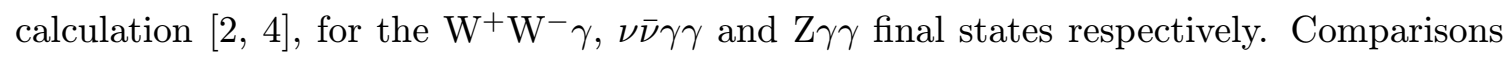
between these programs and the Monte Carlo WRAP [in save shown significant differences for the $\mathrm{Z} \gamma \gamma$ final state; further investigations are in progress in order to clarify this disagreement.

\section{2. $\mathrm{W}^{+} \mathrm{W}^{-} \gamma$ and $\nu \bar{\nu} \gamma \gamma$ production}

At the tree level, many diagrams contribute to the process $\mathrm{e}^{+} \mathrm{e}^{-} \rightarrow \mathrm{W}^{+} \mathrm{W}^{-} \gamma \rightarrow \mathrm{f}_{1} \overline{\mathrm{f}}_{2} \mathrm{f}_{3} \overline{\mathrm{f}}_{4} \gamma$. These diagrams correspond to initial and final state photon radiation, photon radiation from $\mathrm{W}$ bosons and the QGC diagram. The $\mathrm{W}^{+} \mathrm{W}^{-} \gamma$ signal is defined with phase-space cuts, listed in Table 1 , on the photon energy, $E_{\gamma}$, the photon polar angle, $\theta_{\gamma}$, the angle between the direction of the photon and that

\begin{tabular}{|c||c|c|}
\hline & L3 & OPAL \\
\hline \hline$E_{\gamma}$ & $>5 \mathrm{GeV}$ & $>10 \mathrm{GeV}$ \\
$\left|\cos \theta_{\gamma}\right|$ & $<0.94$ & $<0.9$ \\
$\cos \alpha_{\gamma}$ & $<0.94$ & $<0.9$ \\
$M_{\mathrm{f}^{\prime}}$ & - & $>73 \mathrm{GeV}$ \\
\hline
\end{tabular}

Table 1: Signal definition for the process $\mathrm{e}^{+} \mathrm{e}^{-} \rightarrow \mathrm{W}^{+} \mathrm{W}^{-} \gamma \rightarrow \mathrm{f}_{1} \overline{\mathrm{f}}_{2} \mathrm{f}_{3} \overline{\mathrm{f}}_{4} \gamma$. of the closest charged fermion, $\alpha_{\gamma}$, and the invariant mass of the final state fermions, $M_{\mathrm{f} \overline{\mathrm{f}}^{\prime}}$. The analysis of the $\mathrm{e}^{+} \mathrm{e}^{-} \rightarrow \mathrm{W}^{+} \mathrm{W}^{-} \gamma$ process was performed using data collected with OPAL detector at centre-of-mass energy $\sqrt{s}=189$ 


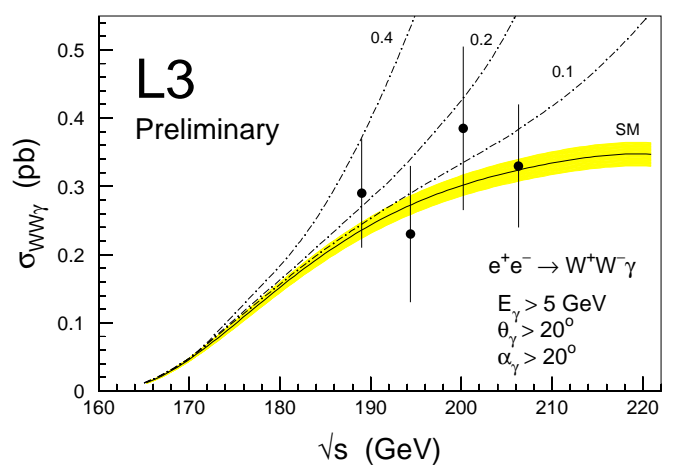

Figure 2: Measured and Standard Model cross section for the process $\mathrm{e}^{+} \mathrm{e}^{-} \rightarrow \mathrm{W}^{+} \mathrm{W}^{-} \gamma$ as a function of $\sqrt{s}$, within the indicate phase-space cuts. The dashed lines correspond to the cross section for non-zero values of the anomalous coupling $a_{n} / \Lambda^{2}$ (in $\mathrm{GeV}^{-2}$ units).

$\mathrm{GeV}$ corresponding to an integrated luminosity $\mathcal{L}=183 \mathrm{pb}^{-1}[\overline{\mathbf{6}}]$ ] at $\sqrt{s}$ between $189 \mathrm{GeV}$ and $209 \mathrm{GeV}$ corresponding to $\mathcal{L} \simeq 600 \mathrm{pb}^{-1}[\mathrm{i}]$. Figure 2 shows the results for the measurement of the cross section from L3 together with the predicted cross section as a function of $\sqrt{s}$. The OPAL analysis yields $\sigma_{\mathrm{WW} \gamma}=0.136 \pm 0.037 \pm$ $0.008 \mathrm{pb}$, in agreement with Standard Model expectations $(0.085 \mathrm{pb}-0.102 \mathrm{pb})$.

The $\mathrm{e}^{+} \mathrm{e}^{-} \rightarrow \nu \bar{\nu} \gamma \gamma$ process was analysed in data collected with the ALEPH $(189 \mathrm{GeV}<$ $\left.\sqrt{s}<209 \mathrm{GeV}, \mathcal{L} \simeq 640 \mathrm{pb}^{-1}\right), \mathrm{L} 3\left(189 \mathrm{GeV}<\sqrt{s}<209 \mathrm{GeV}, \mathcal{L} \simeq 600 \mathrm{pb}^{-1}\right)$ and OPAL $\left(\sqrt{s}=189 \mathrm{GeV}, \mathcal{L} \simeq 180 \mathrm{pb}^{-1}\right)$ detectors.

The selections demand acoplanar photons pairs in events with missing energy and missing trans-

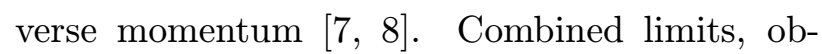
tained by varying one parameter at the time, on anomalous charged QGC parameters at $95 \%$ C.L. are listed in Table 2.

\begin{tabular}{|c||c|}
\hline$a_{0} / \Lambda^{2}\left(\mathrm{GeV}^{-2}\right)$ & $(-0.018,0.018)$ \\
\hline$a_{c} / \Lambda^{2}\left(\mathrm{GeV}^{-2}\right)$ & $(-0.033,0.047)$ \\
\hline$a_{n} / \Lambda^{2}\left(\mathrm{GeV}^{-2}\right)$ & $(-0.17,0.15)$ \\
\hline
\end{tabular}

Table 2: Experimental combined limits on anomalous charged QGC parameters.

\section{3. $\mathrm{Z} \gamma \gamma$ production}

The $\mathrm{e}^{+} \mathrm{e}^{-} \rightarrow \mathrm{Z} \gamma \gamma$ process occurs via radiation of photons from the initial state and is defined by the phase-space requirements, similar to those used in the $\mathrm{W}^{+} \mathrm{W}^{-} \gamma$ final state, listed in Table 3. Only hadronic decays of the $\mathrm{Z}$ boson were considered. These measurements use data collected with the L3 detector $\left(130 \mathrm{GeV}<\sqrt{s}<209 \mathrm{GeV}, \mathcal{L} \simeq 720 \mathrm{pb}^{-1}\right)\left[\begin{array}{l}\overline{9} \\ 9\end{array}\right]$ and with OPAL detector $\left(130 \mathrm{GeV}<\sqrt{s}<209 \mathrm{GeV}, \mathcal{L} \simeq 580 \mathrm{pb}^{-1}\right)$ [1] for the measurement of the cross section, together with the Standard Model predictions, as a function of $\sqrt{s}$. Two-parameters $95 \%$ C.L. limits on the anomalous neutral parameters are reported in Table 4.

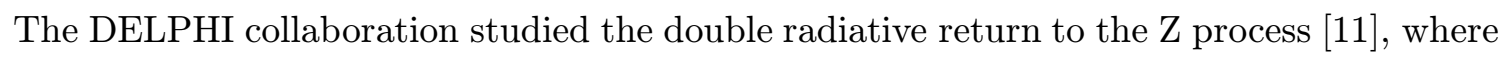
the two emitted photons have similar energies, at $\sqrt{s}$ ranging from $189 \mathrm{GeV}$ to $209 \mathrm{GeV}$ 
$\left(\mathcal{L} \simeq 610 \mathrm{pb}^{-1}\right)$. Only the hadronic decays of the $\mathrm{Z}$ boson were considered. Supposing the photons back-to-back, it holds:

$$
E_{\gamma 1}=\sqrt{s} / 2-M_{\mathrm{Z}}^{2} /\left(2 \sqrt{s}-4 E_{\gamma 2}\right)
$$

where $\mathrm{M}_{\mathrm{Z}}$ is the $\mathrm{Z}$ boson mass, $E_{\gamma 1}$ and $E_{\gamma 2}$ are the energies of the two photons. The signal is then defined by requiring that the most energetic photon of the event has an energy lower than

$$
E_{\gamma 1}^{\max }=\left(s-\left(M_{\mathrm{Z}}+10 \mathrm{GeV}\right)^{2}\right) / 2 \sqrt{s}
$$

and the second one has energy larger than $E_{\gamma 2}^{\min }$, defined by Equation $(\overline{\bar{\beta}} \overline{1} \overline{1})$ for $E_{\gamma 1}=E_{\gamma 1}^{\max }$. In the Equation ( width of the $\mathrm{Z}$ boson. Moreover, it is required that the difference between the photons polar angles exceeds 90 degrees. Three different topologies of events are studied: when both photons are detected, similar to the $\mathrm{Z} \gamma \gamma$ process discussed above, when one photon is not detected because produced at low angle and when both photons go undetected. The results for the measured cross section are summarised in Figure 4.

\begin{tabular}{|c||c|c|}
\hline & L3 & OPAL \\
\hline \hline$E_{\gamma}$ & $>5 \mathrm{GeV}$ & $>5 \mathrm{GeV}$ \\
$\left|\cos \theta_{\gamma}\right|$ & $<0.97$ & $<0.95$ \\
$\cos \alpha_{\gamma}$ & $<0.98$ & $<0.9$ \\
$M_{\mathrm{q} \overline{\mathrm{q}}}$ & $\left(M_{\mathrm{Z}}-2 \Gamma_{\mathrm{Z}}, M_{\mathrm{Z}}+2 \Gamma_{\mathrm{Z}}\right)$ & $80 \mathrm{GeV}-120 \mathrm{GeV}$ \\
\hline
\end{tabular}

Table 3: Signal definition for the process $\mathrm{e}^{+} \mathrm{e}^{-} \rightarrow \mathrm{Z} \gamma \gamma \rightarrow \mathrm{q} \overline{\mathrm{q}} \gamma \gamma$.
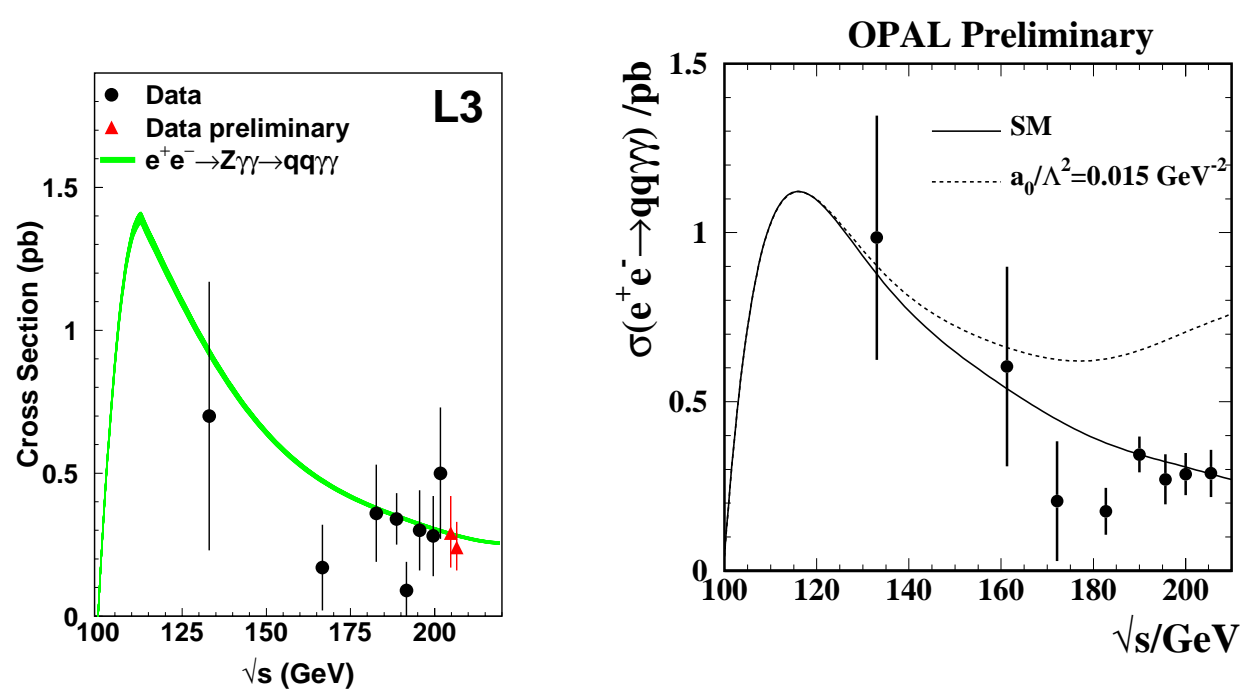

Figure 3: Measured and expected cross section for the process $\mathrm{e}^{+} \mathrm{e}^{-} \rightarrow \mathrm{Z} \gamma \gamma \rightarrow \mathrm{q} \overline{\mathrm{q}} \gamma \gamma$ as a function of $\sqrt{s}$. Different signal definition are used. The dotted line correspond to the cross section for non-zero values of the anomalous couplings. 


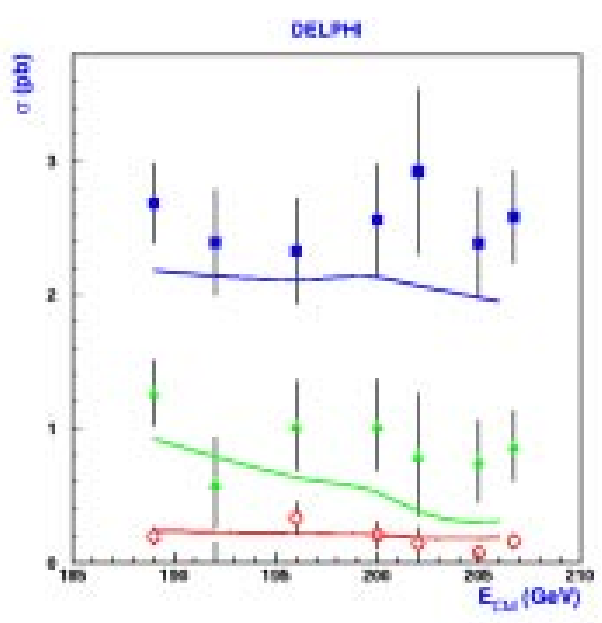

Figure 4: Measured and expected cross sections for the double radiative return to the $\mathrm{Z}$, $\mathrm{e}^{+} \mathrm{e}^{-} \rightarrow \mathrm{Z}(\gamma \gamma) \rightarrow \mathrm{q} \overline{\mathrm{q}}(\gamma \gamma)$, as a function of $\sqrt{s}$, for the three topologies analysed. The uncertainties are statistical only.

\begin{tabular}{|c||c|c|}
\hline & L3 & OPAL \\
\hline$a_{0} / \Lambda^{2}\left(\mathrm{GeV}^{-2}\right)$ & $(-0.008,0.005)$ & $(-0.006,0.008)$ \\
\hline$a_{c} / \Lambda^{2}\left(\mathrm{GeV}^{-2}\right)$ & $(-0.007,0.011)$ & $(-0.008,0.012)$ \\
\hline
\end{tabular}

Table 4: Experimental limits on anomalous neutral QGC parameters.

\section{References}

[1] G. Belanger and F. Boudjema, 'Nucl. Phys. B $288 \overline{1}(199 \overline{2}) \overline{2} \overline{1}$

[2] J.W. Stirling and A. Werthenbach, 'Eur. Phys. J. $\mathbf{C} \overline{1} \overline{4}(\overline{2} \overline{0} \overline{0}) \overline{10} \overline{3}$

[3] F. Piccinini, These Proceedings.

[4] W. J. Stirling and A. Werthenbach, Phys. Lett. B $\mathbf{4} 6 \mathbf{6} 6(1999) 369_{1}^{\prime}$

[5] G. Montagna, M. Moretti, O. Nicrosini, M. Osmo and F. Piccinini, 'P $19 \overline{\vec{T}_{1}}$

[6] The OPAL Collaboration, G. Abbiendi et al., Phys. Lett. B

[7] The L3 Collaboration, L3 note 2675 Contributed paper for International Europhysics Conference on High Energy Physics, July 12-18, 2001, Budapest, Hungary

[8] The ALEPH Collaboration, Contributed paper for International Europhysics Conference on High Energy Physics, July 12-18, 2001, Budapest, Hungary

[9] The L3 Collaboration, * ${ }^{2} 3$ note 2697, Contributed paper for International Europhysics Conference on High Energy Physics, July 12-18, 2001, Budapest, Hungary, 'P $\bar{P} \bar{h}$ s. Lett. Bi :-

[10] The OPAL Collaboration, Physics Notes PN452, 2000

[11] The DELPHI Collaboration, DELPHI 2001-106 CONF 535, Contributed paper for International Europhysics Conference on High Energy Physics, July 12-18, 2001, Budapest, Hungary 